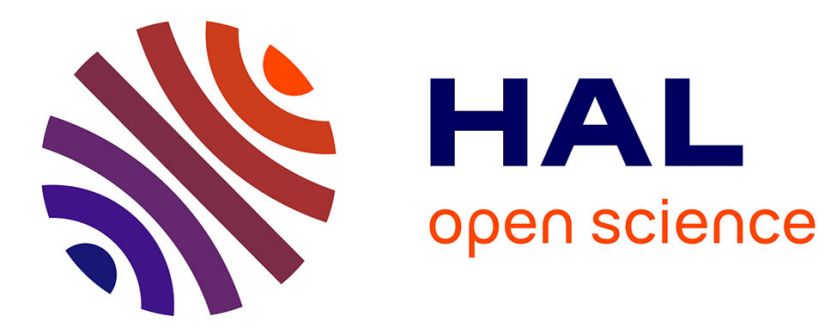

\title{
Identification of protective actions to reduce the vulnerability of safety-critical systems to malevolent acts: a sensitivity-based decision-making approach
}

Tairan Wang, Nicola Pedroni, Enrico Zio

\section{- To cite this version:}

Tairan Wang, Nicola Pedroni, Enrico Zio. Identification of protective actions to reduce the vulnerability of safety-critical systems to malevolent acts: a sensitivity-based decision-making approach. Reliability Engineering and System Safety, 2016, 147, pp.9-18. 10.1016/j.ress.2015.09.005 . hal01270677

\section{HAL Id: hal-01270677 \\ https://hal.science/hal-01270677}

Submitted on 8 Feb 2016

HAL is a multi-disciplinary open access archive for the deposit and dissemination of scientific research documents, whether they are published or not. The documents may come from teaching and research institutions in France or abroad, or from public or private research centers.
L'archive ouverte pluridisciplinaire HAL, est destinée au dépôt et à la diffusion de documents scientifiques de niveau recherche, publiés ou non, émanant des établissements d'enseignement et de recherche français ou étrangers, des laboratoires publics ou privés. 


\title{
Identification of protective actions to reduce the
}

\section{vulnerability of safety-critical systems to malevolent acts: a} sensitivity-based decision-making approach

\author{
Tai-Ran WANG ${ }^{\mathrm{a}}$, Nicola PEDRONI ${ }^{\mathrm{a}}$, Enrico ZIO $\mathrm{ZI}^{\mathrm{a}, \mathrm{b}}$ \\ a Chair on Systems Science and the Energy challenge, Fondation EDF, Ecole Centrale Paris and \\ Supelec, Grande Voie des Vignes, F92-295, Chatenay Malabry Cedex \\ b Politecnico di Milano, Energy Department, Nuclear Section, clo Cesnef, via Ponzio 33/A , 20133, \\ Milan, Italy, Fax: 39-02-2399.6309, Phone: 39-02-2399.6340,enrico.zio@polimi.it
}

\begin{abstract}
A classification model based on the Majority Rule Sorting method has been previously proposed by the authors to evaluate the vulnerability of safety-critical systems (e.g., nuclear power plants) with respect to malevolent intentional acts.

In this paper, we consider a classification model previously proposed by the authors based on the Majority Rule Sorting method to evaluate the vulnerability of safety-critical systems (e.g., nuclear power plants) with respect to malevolent intentional acts. The model is here used as the basis for solving an inverse classification problem aimed at determining a set of protective actions to reduce the level of vulnerability of the safety-critical system under consideration.

To guide the choice of the set of protective actions, sensitivity indicators are originally introduced as measures of the variation in the vulnerability class that a safety-critical system is expected to undergo after the application of a given set of protective actions. These indicators form the basis of an algorithm to rank different combinations of actions according to their effectiveness in reducing the safety-critical systems vulnerability. Results obtained using these indicators are presented with regard to the application of: (i) one identified action
\end{abstract}


at a time, (ii) all identified actions at the same time or (iii) a random combination of identified actions. The results are presented with reference to a fictitious example considering nuclear power plants as the safety-critical systems object of the analysis.

KEYWORDS: safety-critical system, malevolent intentional attacks, vulnerability analysis, protective actions, Majority Rule Sorting (MR-Sort), classification model, inverse classification problem, sensitivity indicator

\section{Notations}

$\operatorname{crit}^{j}$

$x_{i}$

$N P P_{i}$

$C_{i}$

$a c t^{k}$

$\operatorname{coeff} f^{k j}$

$c r i t^{\prime j}$

$B$

$N \uparrow$

$\frac{N \uparrow}{N^{\prime}}$

$N \downarrow$

$\frac{N \downarrow}{N^{\prime}}$

$\Delta N=\frac{N \uparrow}{N^{\prime}}-\frac{N \downarrow}{N^{\prime}}$

$\Delta M \uparrow$ subcriterion $\mathrm{j}$

main criterion $\mathrm{i}$

Nuclear power plant i

vulnerability category $\mathrm{i}$

protective action $\mathrm{k}$

weight of the influence of action $k$ on attribute $j$

after action subcriterion $\mathrm{j}$

limited budget

number of NPPs that are improved after the action(s)

estimate of the percentage of new NPPs that can be

expected to be improved

number of NPPs that are expected to be deteriorated after

the action(s)

estimate of the percentage of new NPPs that can be

expected to be deteriorated

expected "net" amount of ameliorated NPPs

total variation of category underwent by the ameliorated 


$$
\frac{\Delta M \uparrow}{N^{\prime}}
$$

$\Delta M \downarrow$

$\frac{\Delta M \downarrow}{N^{\prime}}$

$\Delta S \uparrow$

$\overline{\Delta M}=\frac{\Delta M \uparrow}{N^{\prime}}-\frac{\Delta M \downarrow}{N^{\prime}}$

$l_{i}^{j}$ "net" variation in vulnerability category that a newly

analyzed NPP is expected to undergo.

ratio between the sums of the variations of vulnerability

category underwent by the ameliorated NPPs and the sum of the corresponding maximum possible category variations

variation in vulnerability category that a new ameliorated plant is expected to undergo

total variation of category underwent by the deteriorated

NPPs

variation in vulnerability category that a new deteriorated plant is expected to undergo

level of action $\mathrm{j}$ applied on system $\mathrm{i}$

\section{INTRODUCTION}

The vulnerability of safety-critical systems and infrastructures (e.g., nuclear power plants) is of great concern, given the multiple and diverse hazards that they are exposed to (e.g., intentional, random, natural etc.) [1] and the potential large-scale consequences. This justifies the increased attention for analyses aimed at (i) the systematic identification of the sources of system vulnerability, (ii) the qualitative and quantitative assessment of system vulnerability [2][3] and (iii) the definition of effective actions of vulnerability reduction.

In a previous work [6], we have proposed an empirical classification framework to tackle the issue (ii) of assessing vulnerability to malevolent intentional acts. Specifically, we have 
adopted a classification model based on the Majority Rule Sorting (MR-Sort) method [7] to assign an alternative (i.e., a safety-critical system) to a given (vulnerability) class (or category). The MR-Sort classification model contains a group of (adjustable) parameters that are calibrated by means of a set of empirical classification examples (also called training set), i.e., a set of alternatives with the corresponding pre-assigned vulnerability classes [6][7]. For further details on this method, the interested reader can refer to the Appendix A at the end of the paper. It is worth mentioning that other majority rule voting methods are widely used in technical decision making problems for vulnerability analysis of systems, see, e.g., [21].

In this paper, we are still only concerned with intentional hazards (i.e., those related to malevolent acts) and address issue (iii) above (i.e., the definition of the actions to undertake for reducing the level of system vulnerability). This issue is difficult to be resolved by traditional risk assessment methods [1][4][5]. On the contrary, the base model developed in Ref. [6] can be extended to address the problem relates to the problem of optimal risk reduction, e.g. by optimization of protective measures [29][30][31]. In other words, an inverse classification problem [8][9][10] of determining a set of protective actions that can effectively reduce the level of vulnerability of a safety-critical system [11], taking into account a specified set of constraints (e.g., budget limits) [8].

The present analysis can be considered part of an encompassing business process of safety management (see, e.g., [22]), where we seek for the best compromise among risks, costs and benefits in allocating investments in safety-critical systems in the presence of uncertainties [28]. Correspondingly, the presented algorithms can be considered part of an encompassing business process of safety management [22]. Mathematically speaking, the aim is to identify how to modify some features of the input patterns (i.e., the attributes of the safety-critical system under analysis) such that the resulting class is changed as desired (i.e., the vulnerability category is reduced to a desired level). To achieve this objective, novel sensitivity indicators [12] are introduced for quantifying the variation in the vulnerability class of a safety-critical system resulting from the application of a given set of protective actions [13]. Using these indicators as the basis for a ranking algorithm, changes in system 
vulnerability can be achieved considering: (i) one identified action at a time, (ii) all identified actions at the same time or (iii) a random combination of identified actions. The proposed indicators also allow different combinations of actions to be ranked and their effectiveness in reducing the vulnerability under specified budget constraints can be evaluated on a new (test) set of (unknown) safety-critical systems (i.e., systems not used before to calibrate/train the classification model). In this context, it is known that existing risk assessment methodologies may fail to account for unknown and emergent risks that are typical of large-scale infrastructure investment allocation problems. On the other hand, in modern portfolio theory, it is well known that a diversified portfolio can be very effective to reduce non-systematic risks. The approach of diversification is equally important in choosing robust portfolios of infrastructure projects that may be subject to emergent and unknown risks [27]. The proposed methodology is expected to contribute also in this direction of optimal classification of options/investments and combinations of the same.

The remainder of the paper is structured as follows. Section 2 recalls the modeling framework for the analysis of vulnerability to intentional hazards. With reference to that, Section 3 introduces the problem of inverse classification. Section 4 describes the sensitivity analysis indicators introduced to tackle the inverse classification problem of Section 3. Section 5 illustrates their use for the identification of protective actions. In Section 6, a case study is proposed to show the application of the method. Finally, Section 7 gives the discussion and conclusions of this research.

\section{THE CLASSIFICATION MODEL FOR THE ASSESSMENT OF VULNERABILITY TO INTENTIONAL HAZARDS}

We limit the vulnerability analysis of a system to the evaluation of the susceptibility to intentional hazards and adopt the three-layers hierarchical model developed in [6] (Figure 1). The susceptibility to intentional hazards (level 1 in Figure 1) is characterized in terms of attractiveness and accessibility (level 2 in Figure 1). These attributes are hierarchically broken 
down into factors which influence them, including resilience interpreted as pre-attack protection (which influences on accessibility) and post-attack recovery (which influences on attractiveness). The disaggregation is made in 6 criteria (level 3 in Figure 1): physical characteristics (x1), social criticality (x2), possibility of cascading failures (x3), recovery means (x4), human preparedness (x5) and level of protection (x6). These six criteria are further decomposed into a layer of $\mathrm{m}=16$ basic subcriteria $\left\{\right.$ crit $\left.^{j}, j=1,2, \ldots, m=16\right\}$ (level 4 in Figure 1), for which data and information are collected in terms of quantitative values or linguistic terms depending on the nature of the subcriterion. The descriptive terms and/ or values of the fourth layer subcriteria are, then, scaled to numerical categories. The criteria included in the layers are defined and assigned "preference directions" for treatment in the decision-making process. The preference direction for a given criterion (e.g., a physical characteristic or parameter of the system) indicates the state towards which it is desirable to "move the parameter" in order to reduce system susceptibility: in other words, the preference direction is assigned from the point of view of a "defender" who is concerned with protecting the system from an attack [16]. Finally, to get the value of the six third-layer criteria $\left\{x_{i}, i=1,2, \ldots, 6\right\}$, (i) we assign weights to each subcriterion to indicate its importance and (ii) we apply a simple weighted sum to the categorical values of the constituent subcriteria $\left\{\right.$ crit $\left.^{j}, j=1,2, \ldots, m=16\right\}$. These $m=16$ criteria $\left\{\right.$ crit $\left.^{j}, j=1,2, \ldots, m=16\right\}$ are evaluated to assess the vulnerability of a given safety-critical system of interest (e.g., a nuclear power plant - NPP).

For the purposes of the present analysis, $M=4$ levels (or categories) of system vulnerability $\{$ Class $=C, C=1,2,3,4\}$ are considered: $1=$ satisfactory, $2=$ acceptable, $3=$ problematic and $4=$ serious.

Then, the assessment of vulnerability corresponds to a classification problem: given the definition of the characteristics of a critical system in terms of the sixteen criteria above, assign the vulnerability category (or class) to which the system belongs.

The classification model is based on the Majority Rule Sorting (MR-Sort) method [7][14][15]; the model contains a group of (adjustable) parameters that have to be calibrated 
by means of a set of empirical classification examples (the training set), i.e., a set of alternatives with the corresponding pre-assigned vulnerability classes. Further details about the classification model are not reported here for brevity: the interested reader is referred to $[16]$.

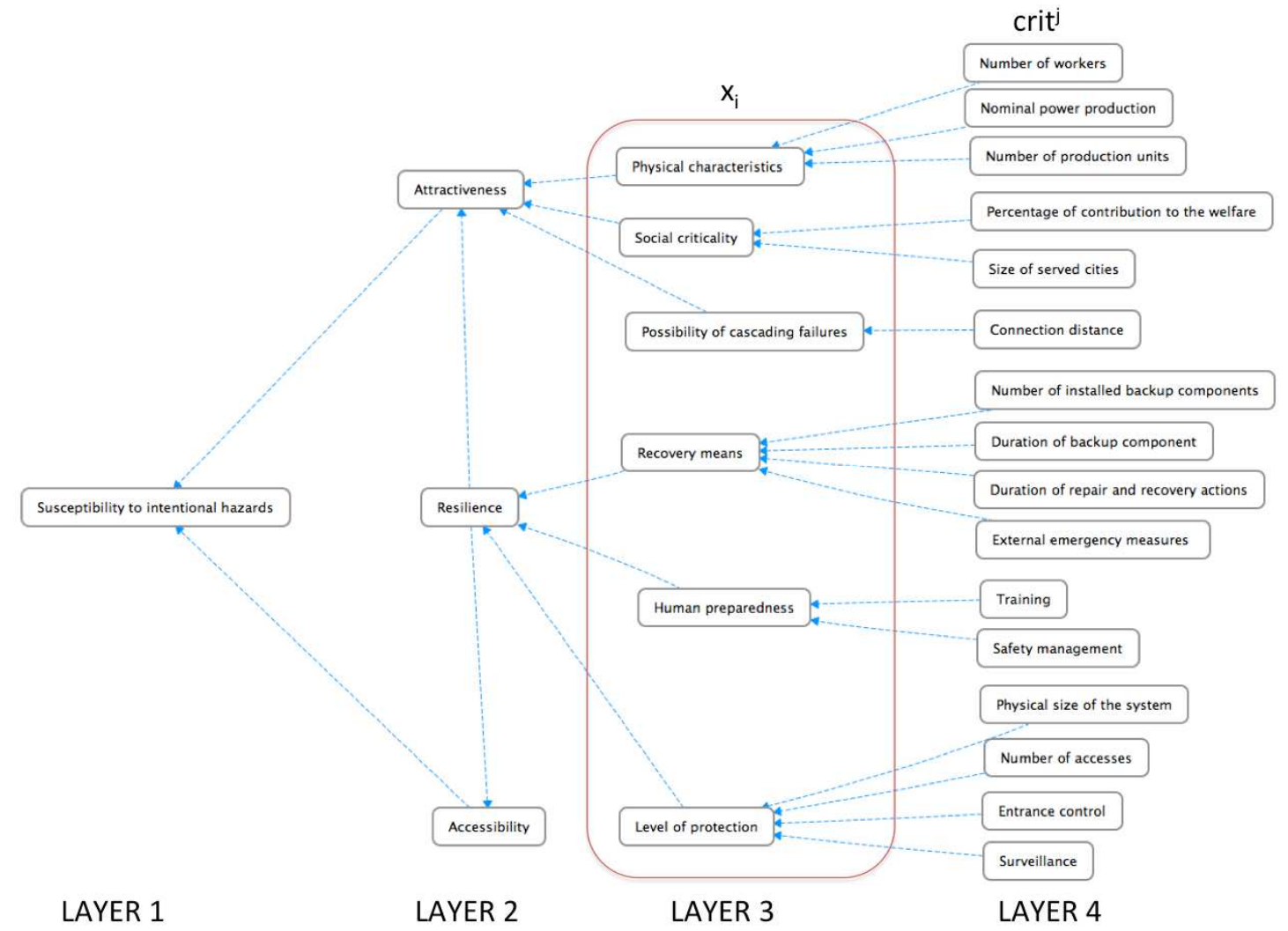

Figure 1. Hierarchical model for susceptibility to intentional hazards [16]

\section{INVERSE CLASSIFICATION PROBLEM FOR PROTECTIVE}

\section{ACTIONS IDENTIFICATION}

We define an inverse classification problem aimed at finding a combination of actions reducing the vulnerability of a (group of) safety-critical system(s) eventually under budget limitations. 


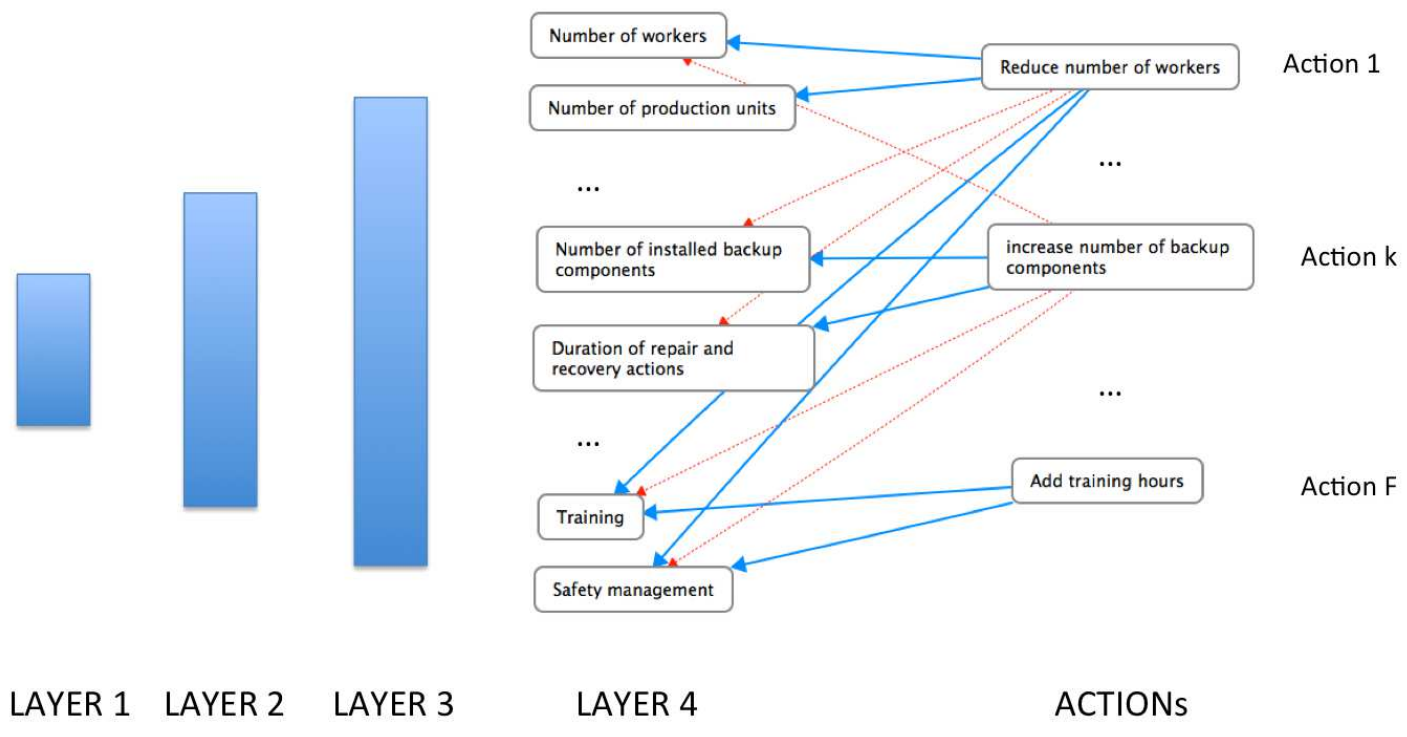

Figure 2. Schema of direct actions for basic criteria

To illustrate the methodology, we consider a set of $N$ NPPs $\left(N P P_{i}, i \in\{1,2, \ldots, N\}\right)$ characterized by $m=16$ basic features $\left(c r i t^{j}, j \in\{1,2, \ldots, m\}\right)$. On the basis of these $m=16$ features, the NPPs are assigned to $M=4$ pre-defined categories $\left(C_{i} \in\{1,2, \ldots, M\}, i \in\{1,2, \ldots, N\}\right)$, where $C_{i}=1$ represents the best situation, i.e., lowest vulnerability. Let $a c t=\left\{a c t^{1}, a c t^{2}, \ldots, a c t^{F}\right\}$ denote the available set of actions, each of which can influence one or more basic criteria crit $^{j}, j \in\{1,2, \ldots, m\}$ (Figure 2) with different intensity, as measured by a set of coefficients $\left\{c o e f f^{k j}, k \in\{1,2, \ldots, F\}, j \in\{1,2, \ldots, m\}\right\}$. In other words, coeff $f^{k j}$ is the "weight" of the influence of action $k$ on attribute $j$ (the higher the absolute value of coef $f^{k j}$, the stronger the effect of action $k$ on attribute $j$ ). Notice that a positive (resp. negative) coefficient coe $f f^{k j}$ means that action $k$ has an ameliorative, positive (resp. deteriorative, negative) effect on attribute $j$, that is, it changes the corresponding value towards (resp. away from) the "preference direction" of attribute $j$; on the contrary, if $c o e f f^{k j}$ is equal to zero, then criterion $j$ is not influenced by action k. "Negative" relationships objectively exist. Actually, taking one action to improve the performance of one specific criterion may lead to a "negative" change in some of the others. For example, increasing the number of backup components on site may lead to an increased number of workers to operate and maintain them, which may increase the possibility of a larger number of injuries of the people exposed to an attack. If the analyst who 
builds the inverse classification model were not able to identify and quantify these "negative" connections (i.e., the coefficients $c o e f f^{k j}$ ), then the (positive) effect of a given combination of actions on a system could be overestimated, with serious drawbacks on the process of resources allocation for system protection.

Significant efforts have been made to assign numerical values to the impacts of actions, in order to represent the problem as realistically as possible. However in a non-fictitious situation, the task is expected to be more complex. Actually, the relations between the actions and the criteria taking into account the dependencies of different attributes and systems are always difficult to identify: in such cases, resorting to the judgment of real experts and possibly to real historical data will be mandatory.

The implementation of one or more actions modifies the attribute values ${ } r i t^{j}, j \in\{1,2, \ldots, m\}$ and as a result, the vulnerability of the system (i.e., the assignment by the classification model) may change. In this paper, we assume that the total effect of the available set of actions $a c t=\left\{a c t^{1}, a c t^{2}, \ldots, a c t^{F}\right\}$ on criterion $j$ is obtained by a linear superposition of the effects of each action $a c t^{i}$ :

$$
\begin{gathered}
\text { crit }^{\prime j}=\operatorname{crit}^{j}+\sum_{k=1}^{F} \operatorname{coeff} f^{k j} * a c t^{k} ; \\
k \in\{1,2, \ldots, F\}, j \in\{1,2, \ldots, m\} .
\end{gathered}
$$

where $c r i t^{\prime j}$ is the value of attribute $j$ after the identified set of available actions has been implemented.

Also, let $\operatorname{Cost}\left(N P P_{i}, a c t^{\prime}\right), a c t^{\prime} \subseteq a c t$ denote the cost of the combination of actions act $t^{\prime}$ applied to $N P P_{i}$. The inverse classification problem can then be formulated as follows: identify the set of actions $\left.a c t_{i}^{\prime} \subseteq a c t\right), i=1,2, \ldots, N$ that improve the vulnerability of the system to a demanded vulnerability category $C_{i}^{\lambda}$ while minimizing the cost, i.e.,

$$
\begin{array}{r}
\min \left(\sum_{i=1}^{N} \operatorname{Cost}\left(N P P_{i}, a c t_{i}^{\prime}\right)\right), a c t_{i}^{\prime} \subseteq a c t ; \\
\text { s.t. } \operatorname{classify}\left(N P P_{i}, a c t_{i}^{\prime}\right)=C_{i}^{\lambda} ; \\
i \in\{1,2, \ldots, N\} .
\end{array}
$$

Alternatively, if it is known that the budget $B_{i}$ is limited for each plant $N P P_{i}$, the formulation becomes: improve the systems to the best possible vulnerability category 
$C_{i}^{\lambda^{\prime}}, C_{i}^{\lambda^{\prime}} \in\{1,2, \ldots, M\}, i \in\{1,2, \ldots, N\}$, while keeping the cost below the available budget $B$ :

$$
\begin{aligned}
& \max \left(\operatorname{classify}\left(N P P_{i}, \operatorname{act}_{i}^{\prime}\right)\right) ; \\
& \text { s.t. } \operatorname{Cost}\left(N P P_{i}, a c t_{i}^{\prime}\right) \leq B_{i}, a c t_{i}^{\prime} \subseteq a c t \text {; } \\
& i \in\{1,2, \ldots, N\} .
\end{aligned}
$$

To address the inverse classification problem, we adopt a pragmatic approach based on sensitivity analysis [17][18][19], introducing indicators that quantify the variation in the vulnerability class that a safety-critical system is expected to undergo upon implementation of a given set of actions.

\section{SENSITIVITY INDICATORS FOR DRIVING THE INVERSE}

\section{CLASSIFICATION PROBLEM}

We consider the group of $N^{\prime}$ vulnerability-class labeled known (available) safety-critical systems (NPPs) used to train the MR-Sort classification model and study the sensitivity of their categories of vulnerability to the implementation of the available protective actions. We denote the original categories of these NPPs as $C_{i}, C_{i} \in\{1,2, \ldots, M\}, i \in\left\{1,2, \ldots, N^{\prime}\right\}$ and the new categories resulting from the application of a set of protective actions as $C_{i}^{\lambda}, C_{i}^{\lambda} \in\{1,2, \ldots, M\}, i \in\left\{1,2, \ldots, N^{\prime}\right\}$.

Let $N \uparrow$ be the number of NPPs that are improved after the action(s):

$$
\begin{gathered}
N \uparrow=\sum_{i=1}^{N^{\prime}} A_{i}, i \in \mathbb{N} ; \\
A_{i}=1, \text { if } C_{i}>C_{i}^{\lambda} ; \\
A_{i}=0, \text { if } C_{i}<C_{i}^{\lambda} .
\end{gathered}
$$

Then, $\frac{N \uparrow}{N^{\prime}}$ can be interpreted as an estimate of the percentage of new (i.e., different from the ones of the training set) NPPs that can be expected to be improved after such action(s) is (are) implemented on them.

Dually, $N \downarrow$, is the number of NPPs that are expected to be deteriorated after the action(s): 


$$
\begin{aligned}
& N \downarrow=\sum_{i=1}^{N^{\prime}} A_{i}, i \in \mathbb{N} ; \\
& A_{i}=1, \text { if } C_{i}<C_{i}^{\lambda} ; \\
& A_{i}=0, \text { if } C_{i}>C_{i}^{\lambda} .
\end{aligned}
$$

Notice that a "deterioration" (i.e., an increase in the vulnerability category) is possible because some of the actions may have positive effects on some subcriteria but negative effects on some others (see Section 3). Then, $\frac{N \downarrow}{N^{\prime}}$ can be interpreted as an estimate of the percentage of new NPPs (i.e., different from the ones of the training set) that can be expected to be deteriorated after such action(s) is (are) implemented on them.

We consider the quantity $\Delta N=\frac{N \uparrow}{N^{\prime}}-\frac{N \downarrow}{N^{\prime}}$ to combine the effects of both positive and negative influences of the actions in the expected "net" amount of ameliorated NPPs.

Considering that the evaluation framework is based on $M=4$ categories, it seems reasonable to consider not only the number of NPPs that are ameliorated or deteriorated, but also the amount of variation in category of vulnerability of each of them. To this aim, we introduce the following indicators to combine the amount of variation in vulnerability with the number of NPPs whose vulnerability category has changed after the actions.

In particular, $\Delta M \uparrow$ is defined as the total variation of category underwent by the ameliorated NPPs:

$$
\begin{array}{r}
\Delta M \uparrow=\sum_{i=1}^{N^{\prime}}\left(M_{i} * A_{i}\right), i \in \mathbb{N} ; \\
M_{i}=C_{i}-C_{i}^{\lambda} ; \\
A_{i}=1, \text { if } C_{i}>C_{i}^{\lambda} ; \\
A_{i}=0, \text { if } C_{i}<C_{i}^{\lambda} .
\end{array}
$$

Thus, $\frac{\Delta M \uparrow}{N^{\prime}}$ can be interpreted as the variation in vulnerability category that a new ameliorated plant is expected to undergo when the chosen combination of actions is applied. Dually, $\Delta M \downarrow$ is defined as: 


$$
\begin{array}{r}
\Delta M \downarrow=\sum_{i=1}^{N^{\prime}}\left(M_{i} * A_{i}\right), i \in \mathbb{N} ; \\
M_{i}=C_{i}-C_{i}^{\lambda} ; \\
A_{i}=1, \text { if } C_{i}<C_{i}^{\lambda} ; \\
A_{i}=0, \text { if } C_{i}>C_{i}^{\lambda} .
\end{array}
$$

Thus, $\frac{\Delta M \downarrow}{N^{\prime}}$ can be seen as the variation in vulnerability category that a new deteriorated plant is expected to undergo when the chosen combination of actions is applied.

Finally, $\overline{\Delta M}=\frac{\Delta M \uparrow}{N^{\prime}}-\frac{\Delta M \downarrow}{N^{\prime}}$ combines the effects of both positive and negative influences of the actions and it can be seen as the "net" variation in vulnerability category that a newly analyzed NPP is expected to undergo after the application of the given set of actions.

The net expected variation in vulnerability category $\overline{\Delta M}$ quantifies the influence of the actions upon the NPPs. However, this measure does not take into account the original category assignment of the NPPs: for example, in practice there is a difference between taking a NPP from category 4 to 3 and taking it from 2 to 1 , even if the category variation is 1 in both cases. To consider this, we introduce the indicator $\Delta S \uparrow$, defined as the ratio between the sums of the variations of vulnerability category underwent by the ameliorated NPPs and the sum of the corresponding maximum possible category variations (i.e., the sum of the category variations that the NPPs would undergo if they were ameliorated to the best possible vulnerability category):

$$
\begin{array}{r}
\Delta S \uparrow=\frac{\Delta M \uparrow}{E} ; \\
E=\sum_{i=1}^{N^{\prime}}\left(C_{i}-C^{b e s t}\right) * A_{i}, i \in \mathbb{N} ; \\
A_{i}=1, \text { if } C_{i}>C_{i}^{\lambda} ; \\
A_{i}=0, \text { if } C_{i}<C_{i}^{\lambda} .
\end{array}
$$

The indicator $\Delta S \uparrow$ quantifies the influence of the actions on NPPs, relative to their original categories: the lower $\Delta S \uparrow$ is, the higher the influence of the chosen set of actions is on the NPPs originally of a relatively low category.

Based on the above indicators, an algorithm is proposed to rank different combinations of actions according to their effectiveness in reducing the vulnerability of safety-critical systems. 
The actions with positive influences are obviously preferred. On the contrary, concerning the ones with negative influences, the rationality of being chosen as ameliorative actions should be reconsidered. The analyst may replace/modify/delete them from the original considered action set. The algorithm proceeds as follows:

(1) Rank the (combinations of) actions according to the value of $\overline{\Delta M}$ (the higher the value of $\overline{\Delta M}$, the more effective the combination of actions in reducing vulnerability):

- combinations of actions that have a negative value of $\overline{\Delta M}(\overline{\Delta M}<0)$ are expected to increase the vulnerability of a NPP: this is due to the fact that some actions may have a deteriorated effect on some of the subcriteria that more than counter balances the positive effects on their subcriteria. The identification of the combination of actions with $\overline{\Delta M}<0$ allows the analyst to (i) study the mechanisms of influence of the actions on the basic subcriteria (Layer 4 in Figures 1 and 2) and (ii) if possible, eliminate the "negative connections", i.e., the negative dependencies between some actions and some criteria (e.g., by identifying alternative actions for dealing with these "critical" subcriteria);

- the actions that have a positive value of $\overline{\Delta M}(\overline{\Delta M}>0)$ are expected to reduce the vulnerability and are assigned higher rankings (the higher $\overline{\Delta M}$, the higher the ranking);

(2) If several combinations of actions have the same value of $\overline{\Delta M}$, then consider the other indicators (i.e., $\frac{N \uparrow}{N^{\prime}}$ and $\frac{\Delta M \uparrow}{N^{\prime}}$ ): depending on the judgment of the DMs, higher importance may be given to those actions that produce a larger expected number of improved NPPs $\left(\frac{N \uparrow}{N^{\prime}}\right)$ or to those that generate a higher "expected class improvement" $\left(\frac{\Delta M \uparrow}{N^{\prime}}\right)$.

(3) If some combinations still have the same ranking, analyze indicator $\Delta S \uparrow$ to check which actions have stronger impact on the NPPs of low categories. 


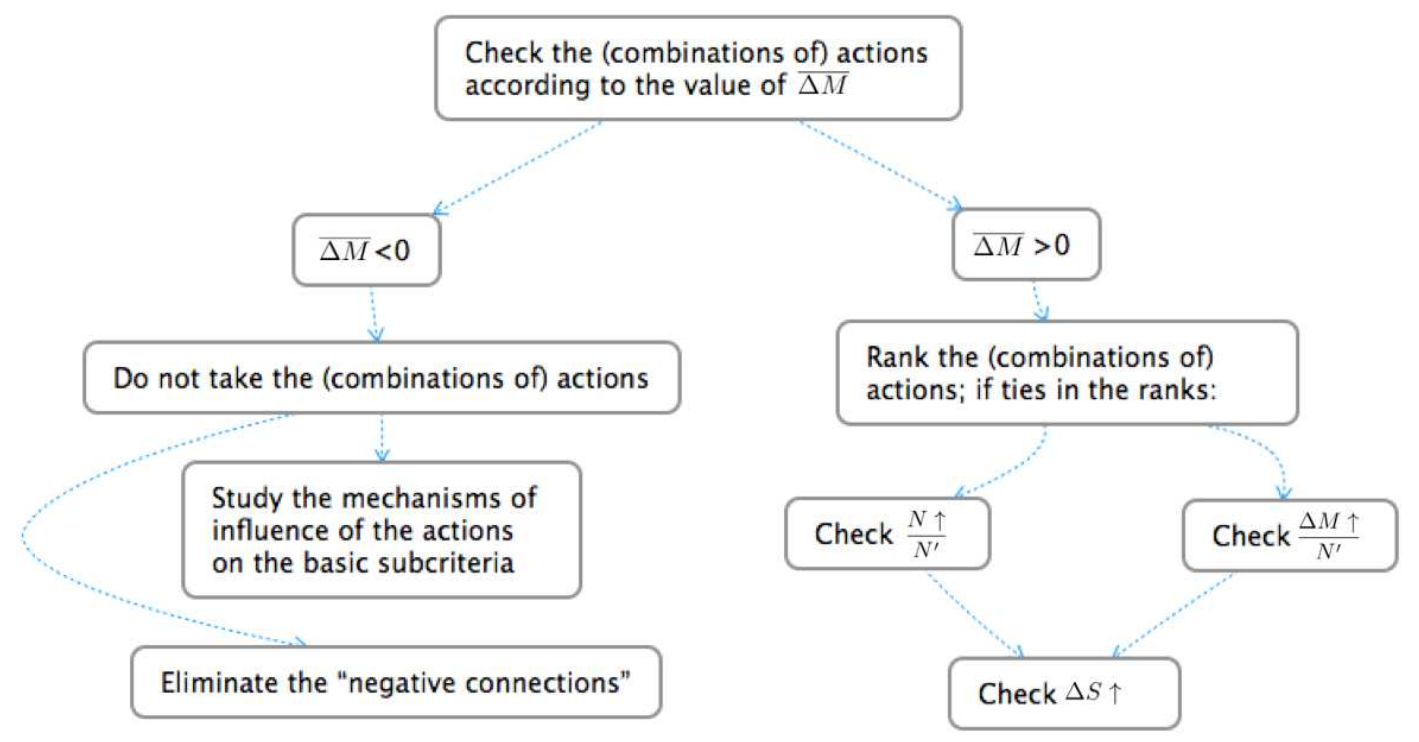

Figure 3. Schema of decision logic for selecting an action

\section{CASE STUDY}

The sensitivity analysis proposed in Section 4 is applied on a case study concerning the vulnerability analysis of NPPs [6]. We refer to the $n=6$ main criteria $i=1,2, \ldots, n=6$ of the hierarchical modeling presented in [6] and recalled in Section 2: physical characteristics $\left(\mathrm{x}_{1}\right)$, social criticality $\left(\mathrm{x}_{2}\right)$, possibility of cascading failures $\left(\mathrm{x}_{3}\right)$, recovery means $\left(\mathrm{x}_{4}\right)$, human preparedness $\left(\mathrm{x}_{5}\right)$ and level of protection $\left(\mathrm{x}_{6}\right)$; these criteria are numbers scaled in the range $[0,1]$. Then, the main criteria are successively broken into a layer of $m=16$ basic criteria (Figure 2). Finally, $\mathrm{M}=4$ vulnerability categories Class $=C, C=1,2,3,4$ are defined as: $1=$ satisfactory, $2=$ acceptable, $3=$ problematic and $4=$ serious $($ Section 2$)$.

As shown in Figure 2 and anticipated in Section 3, we define $\mathrm{F}=13$ direct actions $\left(a c t=\left\{a c t^{1}, a c t^{2}, \ldots, a c t^{F}\right\}\right)$, each acting on one or more subcriteria (Table 1). All the actions have multiple influences on different criteria, with possibly positive or negative effects: for example, the action "reduce the number of workers" has an obvious direct influence on the subcriterion "Number of workers", but may also imply, e.g., (i) reducing the number of production units, the number of accesses to the plant, the number of installed backup components and external emergency measures; (ii) increasing the duration of repair and recovery actions; (iii) enhancing the training; (iv) facilitating the safety management and 
entrancing control and surveillance. The strengths of the influences of the actions on the different criteria are quantified by the different weights/coefficients reported in Table 1.

Also, for each action we consider different levels of implementation $l_{i}^{j}$ ( $\left.l_{i}^{j}, l_{i}^{j} \in\{0,1,2,3\}, i \in\{1,2, \ldots, N\}, j \in\{1,2, \ldots, F\}\right)$, representing to what extent/ how far/ in which amount action $j$ is applied on system $i$ (notice that $l_{i=0}^{j}$ means that action $j$ is not applied to system $i$ ) (Table 1).

Finally, for simplicity we assume that the cost related to the application of a given action is equal to the level $l_{i}^{j}$ of the action: for example, referring to Table 1, if we choose to reduce the number of workers by $20 \%$, the related cost is 1 in arbitrary units (since the action corresponds to level $l_{i=1}^{1}$ ); on the contrary, if we reduce the number of workers by $30 \%$, the

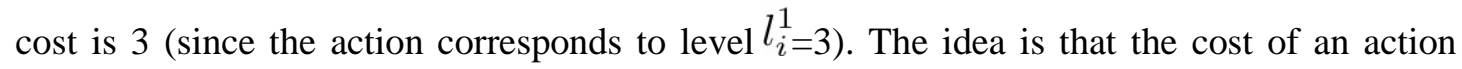
increases (resp. decreases) with its "level" of "strength" of implementation. Notice that however, the levels assigned to the actions are not always strictly "mathematically" proportional to the change of value they produce in the corresponding criteria. In fact, for different actions, the three levels of "effects" on the corresponding directly influenced criteria may be of different notice. Sometimes they may be represented by a quantitative discrete number (e.g., for action "reduce number of production units" we have -1 production unit for level 1, -2 production units for level 2, -3 production units for level 3); sometimes they may be a percentage (as for the number of workers mentioned above). In addition, the costs of an action and the corresponding change in a criterion value are not strictly proportional either (e.g., the cost of training enhance may be the same for 50 and for 80 people, but different for100). In this view, choosing the cost of an action equal to the level lij of implementation of the action is a (maybe rough) compromise between simplicity and pragmatic engineering sense. Obviously, in reality, the costs should be defined in a more sophisticated way and possibly they should be different for different levels of different actions towards different criteria.

Table 1. Available actions and coefficients of influences of the actions on different subcriteria 


\begin{tabular}{|c|c|}
\hline No. & Action description \\
\hline act $^{1}$ & Reduce number of workers \\
\hline $\mathrm{act}^{2}$ & Reduce nominal power production \\
\hline $\mathrm{act}^{3}$ & Reduce number of production units \\
\hline $\mathrm{act}^{4}$ & Reduce percentage of contribution to the welfare \\
\hline $\operatorname{act}^{5}$ & Increase number of installed backup components \\
\hline $\operatorname{act}^{6}$ & Increase external emergency measures \\
\hline $\mathrm{act}^{7}$ & Increase duration of backup component \\
\hline $\operatorname{act}^{8}$ & Reduce duration of repair and recovery actions \\
\hline act $^{9}$ & Enhance training \\
\hline act $^{10}$ & Enhance safety management \\
\hline $\operatorname{act}^{11}$ & Reduce number of accesses \\
\hline $\operatorname{act}^{12}$ & Enhance entrance control \\
\hline $\operatorname{act}^{13}$ & Enhance surveillance \\
\hline
\end{tabular}

\begin{tabular}{|c|c|c|c|c|c|c|c|c|}
\hline & $\begin{array}{c}\text { Number } \\
\text { of } \\
\text { workers }\end{array}$ & $\begin{array}{c}\text { Nominal } \\
\text { power } \\
\text { production }\end{array}$ & $\begin{array}{c}\text { Number } \\
\text { of } \\
\text { production } \\
\text { units }\end{array}$ & $\begin{array}{c}\text { Percentage } \\
\text { of } \\
\text { contribution } \\
\text { to the } \\
\text { welfare }\end{array}$ & $\begin{array}{c}\text { Size } \\
\text { of } \\
\text { served } \\
\text { cities }\end{array}$ & $\begin{array}{c}\text { Connection } \\
\text { distance }\end{array}$ & $\begin{array}{l}\text { Number of } \\
\text { installed } \\
\text { backup } \\
\text { components }\end{array}$ & $\begin{array}{l}\text { External } \\
\text { emergency } \\
\text { measures }\end{array}$ \\
\hline Actions & Crit1 & Crit2 & Crit3 & Crit4 & Crit5 & Crit6 & Crit7 & Crit8 \\
\hline act $^{1}$ & 1 & 0 & 1 & 0 & 0 & 0 & -0.4 & -1 \\
\hline $\mathrm{act}^{2}$ & 0 & 1 & 0 & 1 & 0 & 0 & 0 & 0 \\
\hline $\mathrm{act}^{3}$ & 0.7 & 1 & 1 & 0 & 0 & 0 & 0.6 & 0 \\
\hline $\mathrm{act}^{4}$ & 0 & 0 & 0 & 1 & -1 & 0 & 0 & 0 \\
\hline $\mathrm{act}^{5}$ & -0.2 & 0 & 0 & 0 & 0 & 0 & 1 & 0 \\
\hline $\mathrm{act}^{6}$ & -0.1 & 0 & 0 & 0 & 0 & 0 & 0 & 1 \\
\hline act $^{7}$ & 0 & 0 & 0 & 0 & 0 & 0 & 0 & 0 \\
\hline $\mathrm{act}^{8}$ & 0 & 0 & 0 & 0 & 0 & 0 & 0 & 0 \\
\hline
\end{tabular}




\begin{tabular}{|c|c|c|c|c|c|c|c|c|}
\hline $\mathrm{act}^{9}$ & 0 & 0 & 0 & 0 & 0 & 0 & 0 & 0 \\
\hline $\mathrm{act}^{10}$ & 0 & 0 & 0 & 0 & 0 & 0 & 0 & 0 \\
\hline $\mathrm{act}^{11}$ & 0 & 0 & 0 & 0 & 0 & 0 & 0 & 0 \\
\hline $\mathrm{act}^{12}$ & 0 & 0 & 0 & 0 & 0 & 0 & 0 & 0 \\
\hline $\mathrm{act}^{13}$ & 0 & 0 & 0 & 0 & 0 & 0 & 0 & 0 \\
\hline
\end{tabular}

\begin{tabular}{|c|c|c|c|c|c|c|c|c|}
\hline & $\begin{array}{l}\text { Duration } \\
\text { of backup } \\
\text { component }\end{array}$ & $\begin{array}{c}\text { Duration of } \\
\text { repair and } \\
\text { recovery } \\
\text { actions }\end{array}$ & Training & $\begin{array}{c}\text { Safety } \\
\text { management }\end{array}$ & $\begin{array}{c}\text { Physical } \\
\text { size of } \\
\text { the } \\
\text { system }\end{array}$ & $\begin{array}{l}\text { Number of } \\
\text { accesses }\end{array}$ & $\begin{array}{c}\text { Entrance } \\
\text { control }\end{array}$ & Surveillance \\
\hline Actions & Crit9 & Crit10 & Crit11 & Crit12 & Crit13 & Crit14 & Crit15 & Crit16 \\
\hline act $^{1}$ & 0 & -0.2 & 0.5 & 0.5 & 0 & 1 & 0.4 & 0.4 \\
\hline $\mathrm{act}^{2}$ & 0 & 0 & 0 & 0.2 & 0 & 0 & 0 & 0 \\
\hline $\mathrm{act}^{3}$ & 0.2 & 0.3 & 0.4 & 0.2 & 0.7 & 0 & 0 & 0.3 \\
\hline $\mathrm{act}^{4}$ & 0 & 0 & 0 & 0.1 & 0 & 0 & 0 & 0 \\
\hline $\mathrm{act}^{5}$ & 0.5 & 0 & -0.2 & 0.1 & 0 & 0 & 0 & -0.15 \\
\hline $\mathrm{act}^{6}$ & 0.3 & 0 & -0.1 & 0.05 & -0.3 & 0 & 0 & -0.05 \\
\hline $\mathrm{act}^{7}$ & 1 & 0 & 0 & 0.1 & 0 & 0 & 0 & 0 \\
\hline $\mathrm{act}^{8}$ & 0 & 1 & -0.2 & 0.1 & 0 & 0 & 0 & 0 \\
\hline $\mathrm{act}^{9}$ & 0 & 0.5 & 1 & 0.2 & 0 & 0 & 0.2 & 0 \\
\hline $\mathrm{act}^{10}$ & 0 & 0 & -0.2 & 1 & 0 & 0 & 0 & 0 \\
\hline $\mathrm{act}^{11}$ & 0 & 0 & -0.1 & 0.1 & 0 & 1 & 0.4 & 0.1 \\
\hline $\mathrm{act}^{12}$ & 0 & 0 & -0.1 & 0.1 & 0 & 0 & 1 & 0 \\
\hline $\mathrm{act}^{13}$ & 0 & 0 & 0 & 0.2 & 0 & 0 & 0 & 1 \\
\hline
\end{tabular}

\begin{tabular}{|c|c|c|c|}
\hline No. & level1 & level2 & level3 \\
\hline $\mathrm{act}^{1}$ & $20 \%$ & $25 \%$ & $30 \%$ \\
\hline $\mathrm{act}^{2}$ & $20 \%$ & $30 \%$ & 3 \\
\hline $\mathrm{act}^{3}$ & 1 & 2 & $30 \%$ \\
\hline $\mathrm{act}^{4}$ & $10 \%$ & 2 & 3 \\
\hline $\mathrm{act}^{5}$ & 1 & 1 & 2 \\
\hline $\mathrm{act}^{6}$ & 0.5 & 24 & 48 \\
\hline $\mathrm{act}^{7}$ & 12 & $20 \%$ & 2 \\
\hline
\end{tabular}




\begin{tabular}{|c|c|c|c|}
\hline act $^{8}$ & 6 & 12 & 24 \\
\hline act $^{9}$ & 1 & 3 & 5 \\
\hline act $^{10}$ & 1 & 3 & 3 \\
\hline act $^{11}$ & 1 & 2 & 3 \\
\hline act $^{12}$ & 1 & 2 & 3 \\
\hline act $^{13}$ & 1 & 2 & 5 \\
\hline
\end{tabular}

In what follows, two analyses are performed: first, based on the indicators of Section 4, different combinations of actions are ranked according to their ability in reducing the vulnerability of a group of NPPs (Section 5.1); then, the inverse classification problem of Section 3 is tackled using the sensitivity indicators of Section 4 and taking into account the action costs and budget limitations (Section 5.2).

\subsection{Ranking different combinations of actions based on $\overline{\Delta M}$}

A set $G$ of $N(N=20)$ NPPs $\left(G=\left\{N P P_{i}, i \in\{1,2, \ldots, N\}\right\}\right)$ is available: 10 of them (NPPs from No.6 to No.15 $\left.G^{\text {ref }}=\left\{N P P_{i}, i \in\{6,7, \ldots, 15\}\right\}\right)$ are selected as a reference set to evaluate the sensitivity indicators; the remaining NPPs are regrouped to form a set $G^{\text {test }}$ ( $\left.G^{\text {test }}=\left\{N P P_{i}, i \in\{1,2, \ldots, 5\} \cup\{16,17, \ldots, N\}\right\}\right)$ used to test the combinations of actions ranked using $G^{r e f}$. Based on the reference set, we have performed an exhaustive calculation of the value of $\overline{\Delta M}$ for all the possible combinations of actions (in total, $4^{\wedge} 13=67108864$ combinations). Then, we selected the ones (in total 29940 combinations) that have the (same) highest value of $\overline{\Delta M}$ (i.e., $\overline{\Delta M}=14$ ): these represent the optimal combinations of actions according to $\overline{\Delta M}$ : in what follows, this set is referred to as Combination $\frac{\text { highest }}{\Delta M}$.

All the combinations of actions belonging to the set Combination $\frac{\text { highest }}{\Delta M}$ are applied to each of the $N(N=20)$ NPPs in $G$ : the resulting categories $\left(C_{i}^{\lambda^{\prime}}, i \in\{1,2, \ldots, N\}\right)$ are reported in Table 2. Note that the actions are ranked according to values of $\overline{\Delta M}$ that are evaluated on a group of reference plants $\left(G^{r e f}\right)$ : in this view, they provide an indication only on the expected performance of the actions on new plants and, thus, they may not provide any indications 
about the combination of actions that is optimal for one particular plant. Thus, in order to verify how close these sets of actions are to the combinations that are optimal for a particular NPP, we compare the assignments $C^{\lambda^{\prime}}$ (Table 2) with the best category that each NPP may reach $\left(C_{i}^{\lambda}, i \in\{1,2, \ldots, N\}\right.$ ) (in other words, $C^{\lambda}$ is the category that $N P P_{i}$ reaches after the application of a combination of actions that is the optimal one for that particular plant). In order to do so, another exhaustive calculation is done upon the group $G$ with the purpose of finding the actions that bring each particular NPP to the best category possible (notice that for some NPPs, reaching category 1 may not be possible). All the possible combinations of actions are tested on each NPP in order to find the best assignment $C_{i}^{\lambda}$ for each of them. The results are shown in Table 2. The first column of the results shows the original assignments for the NPPs in the studied set $G$. The second column shows the corresponding possibly best assignments $C^{\lambda}$ and the third column provides the new assignments $C^{\lambda^{\prime}}$ after the application of the combinations of actions included in Combination $\frac{\text { highest }}{\Delta M}$.

Analyzing the best assignments $C^{\lambda}$ of the NPPs in the reference set $\left(C_{i}^{\lambda}, i \in\{6,7, \ldots, 15\}\right)$, we observe that they coincide perfectly $(100 \%)$ with the assignments $C^{\lambda^{\prime}}\left(C_{i}^{\lambda^{\prime}}, i \in\{6,7, \ldots, 15\}\right)$ obtained after the application of the actions in Combination $\frac{\text { highest }}{\Delta M}$. If we take the NPPs in the test set as new NPPs and compare the assignments obtained by these two methods with the original assignments $C\left(C_{i}, i \in\{1,2, \ldots, 5\} \cup\{16,17, \ldots, N\}\right)$, we find that: (i) all the NPPs are stable or ameliorated after the application of the combinations of actions in Combination $\frac{\text { highest }}{\Delta M}$; (ii) there are 2 out of 10 NPPs that are not ameliorated to the best category $C_{i}^{\lambda}$ (i.e., NPPs 16 and 19): they remain in the same category; instead, 8 out of 10 NPPs are ameliorated to their best possible categories: then, the probability that the combinations of actions Combination $\frac{\text { highest }}{\Delta M}$ ameliorate a new NPP to its best possible category $C^{\lambda}$ is $80 \%$.

Table 2. Comparison of assignments: Best possible Assignment $C_{i}^{\lambda}$ and After action Assignment $C_{i}^{\lambda^{\prime}}$ listed with NPPs that are differently assigned highlighted (NPP16, NPP19) 


\begin{tabular}{|c|c|c|c|}
\hline No. & Original Assignment & $\begin{array}{l}\text { Best possible Assignment } \\
\qquad C_{i}^{\lambda}\end{array}$ & $\begin{array}{l}\text { After action Assignment } \\
\qquad C_{i}^{\lambda \prime}\end{array}$ \\
\hline NPP1 & 1 & 1 & 1 \\
\hline NPP2 & 3 & 3 & 3 \\
\hline NPP3 & 2 & 2 & 2 \\
\hline NPP4 & 3 & 1 & 1 \\
\hline NPP5 & 3 & 2 & 2 \\
\hline NPP6 & 2 & 1 & 1 \\
\hline NPP7 & 2 & 1 & 1 \\
\hline NPP8 & 4 & 2 & 2 \\
\hline NPP9 & 4 & 2 & 2 \\
\hline NPP10 & 4 & 3 & 3 \\
\hline NPP11 & 1 & 1 & 1 \\
\hline NPP12 & 2 & 1 & 1 \\
\hline NPP13 & 3 & 2 & 2 \\
\hline NPP14 & 3 & 1 & 1 \\
\hline NPP15 & 4 & 1 & 1 \\
\hline NPP16 & 3 & 2 & 3 \\
\hline NPP17 & 2 & 2 & 2 \\
\hline NPP18 & 3 & 2 & 2 \\
\hline NPP19 & 3 & 2 & 3 \\
\hline NPP20 & 2 & 1 & 1 \\
\hline
\end{tabular}

5.2 Constrained inverse problem: identification of the best combination of actions considering constraints

In a more realistic case, the cost of the protective actions should be considered. Although in reality the costs of different actions can be different, and the same action may cost differently when applied to different NPPs, for simplicity, in this paper we define the Cost of a combination of actions (in arbitrary units) as the sum of the levels $l_{i}^{j}$ of the actions: 


$$
\begin{aligned}
& \text { Cost }=\sum_{i=1}^{N} \operatorname{Cost}_{i} ; \\
& \text { Cost }_{i}=\sum_{j=1}^{F} l_{i}^{j} * A_{i}^{j} ; \\
& A_{i}=1, \text { if } l_{i}^{j} \neq 0 ; \\
& A_{i}=0, \text { if } l_{i}^{j}=0 ; \\
& i \in\{1,2, .,, N\}, j \in\{1,2, \ldots, F\} .
\end{aligned}
$$

We assume that a budget $B_{i}$ is allocated for the improvement of the generic power plant $N P P_{i}$ : the budgets $B_{i}, i \in\left\{1,2, \ldots, N^{G^{t e s t}}\right\}$ allocated for the NPPs of the test set $G^{\text {test }}=\left\{N P P_{i}, i \in\{1,2, \ldots, 5\} \cup\{16,17, \ldots, N\}\right\}$ are shown in Table 3.

Table 3. Budgets available for the NPPs belonging to the test set $G^{\text {test }}$

\begin{tabular}{|c|c|}
\hline No. & Budget $B_{i}$ \\
\hline NPP1 & 10 \\
\hline NPP2 & 25 \\
\hline NPP3 & 15 \\
\hline NPP4 & 5 \\
\hline NPP5 & 16 \\
\hline NPP16 & 19 \\
\hline NPP17 & 10 \\
\hline NPP18 & 23 \\
\hline NPP19 & 9 \\
\hline NPP20 & 17 \\
\hline
\end{tabular}

As before, we take the reference set $G^{r e f}=\left\{N P P_{i}, i \in\{6,7, \ldots, 15\}\right\}$ to calculate the value of $\overline{\Delta M}$ for all possible combinations of actions. Then, for each NPP in the test set $G^{\text {test }}$, we identify the combination(s) of actions with the highest value of $\overline{\Delta M}$ and whose costs $\operatorname{Cost}_{i}\left(i \in\left\{1,2, \ldots, N^{G^{t e s t}}\right\}\right)$ are lower than or equal to the given budgets $B_{i}$ :

Find act $_{i}$ :

$$
\begin{array}{r}
\operatorname{Max}\left(\overline{\Delta M}\left(N P P_{i}, a c t_{i}\right)\right), \\
a c t_{i} \subseteq a c t ; \\
\text { s.t. } \text { Cost }_{i} \leq B_{i} ; \\
i \in\left\{1,2, \ldots, N^{G^{\text {est }}}\right\} .
\end{array}
$$


The results are shown in Table 4. Among all the possible combinations of actions, the ones that present the highest value of $\overline{\Delta M}\left(\overline{\Delta M}^{\text {Max }}=14\right)$ have a minimum cost $\operatorname{Cost}^{M i n}=19$. So, all the NPPs in the test set $G^{\text {test }}$ that have a budget higher than or equal to $\operatorname{Cost}^{M i n}$ (i.e., NPP2, NPP16 and NPP18) can be ameliorated to their corresponding best possible categories (as presented in Section 4). Five of the remaining NPPs (i.e., NPP1, NPP3, NPP17, NPP19 and NPP20) can still be ameliorated to the same category that would be obtained by the actions in the set act(with Cost $\leq 10,15,10,9,17$ ), even though they have a budget, which is lower than, $\operatorname{Cost}^{M i n}=19$ and a performance lower than $\overline{\Delta M}^{\text {Max }}=14$.

Table 4. Assignments comparison

\begin{tabular}{|c|c|c|c|c|}
\hline No. & $\begin{array}{c}\text { Original } \\
\text { Assignment }\end{array}$ & $\begin{array}{c}\text { Best possible } \\
\text { Assignment }\end{array}$ & Best Assignment & $\begin{array}{c}\text { Limited Budget } \\
\text { Assignment }\end{array}$ \\
\hline NPP1 & 1 & 1 & 1 & 1 \\
\hline NPP2 & 3 & 3 & 3 & 3 \\
\hline NPP3 & 2 & 2 & 1 & 1 or 2 \\
\hline NPP4 & 3 & 1 & 2 & 2 or 3 \\
\hline NPP5 & 3 & 2 & 3 & 2 \\
\hline NPP16 & 3 & 2 & 2 & 2 \\
\hline NPP17 & 2 & 2 & 3 & 1 \\
\hline NPP18 & 3 & 2 & 1 & 3 \\
\hline NPP19 & 3 & 2 & 1 & 2 \\
\hline NPP20 & 2 & & & 2 \\
\hline
\end{tabular}

The situation is different for NPP4 and NPP5 (Table 5). They are originally assigned to category 3. NPP4 can be ameliorated by any combination of actions belonging to act(with Cost $\leq 5)$. Among all the combinations of actions that have the best value of $\overline{\Delta M}$ equal to 7 and cost limited by the given budget, $73.91 \%$ can bring NPP4 up to category 2 and $26.09 \%$ can bring it to the best category. Instead, NPP5 cannot be ameliorated to the best category by any of the combinations: in particular, $18.52 \%$ of the actions leave such NPP in category 3 whereas $81.48 \%$ bring it up to category 2 . 
Table 5. Assignments for NPP4 and NPP5

\begin{tabular}{|c|c|c|c|}
\hline Assignment & 3 & 2 & 1 \\
\hline NPP4 & $0.00 \%$ & $73.91 \%$ & $26.09 \%$ \\
\hline NPP5 & $18.52 \%$ & $81.48 \%$ & $0.00 \%$ \\
\hline
\end{tabular}

\section{CONCLUSIONS}

In this paper, we have developed a pragmatic inverse classification framework for identifying ameliorative action(s) to reduce the vulnerability with respect to intentional hazards of safetycritical systems (in the example of reference, Nuclear Power Plants-NPPs). An MR-sort classification model calibrated on a small-sized set of data representing a priori-known classification examples has been used. Sensitivity indicators have been introduced to evaluate combinations of actions with respect to their ability to reduce the vulnerability of the safetycritical systems considered. A case study referring to NPPs vulnerability to intentional attacks has been worked out. The results show that the actions ranked as best according to the proposed indicators give a satisfactory performance in terms of reduction of vulnerability in test NPPs, even in presence of budget constraints: for example, in the case without budget constraints eight out of ten NPPs are ameliorated to their best possible categories, whereas two of them remain in the same categories; in the constrained case still six of the ten NPPs are brought to their best possible vulnerability classes.

The proposed methodological framework provides a powerful tool for systematically and pragmatically evaluating the safety and vulnerability as well as other characteristics of critical systems.

For future research, the following issues will be considered. Since one set of weights is usually an insufficient basis for giving priorities, the sensitivity of investment priorities to the weights of criteria can be tackle: for example, in [22][23][24] a "scenario" is introduced that reflects a set of weights for each stakeholder, such as emphasis on particular aspects of safety in the aftermath of a major nuclear incident. 
As presented in [25], an influential set of weights can suggest $R \& D$ priorities in protection of energy systems.

Moreover, a set of weights can also be brought by other stakeholders, such as owners, operators and users etc: each set of weights presumably leads to variation in the preferred safety investments [26].

In addition, although in this work significant efforts have been made to assign numerical values to the impacts of actions (in order to represent the problem as realistically as possible), in a non-fictitious situation the task is expected to be more complex. Actually, the relations between the actions and the criteria taking into account the dependencies of different attributes and systems are always difficult to identify: in such cases, resorting to the judgment of real experts and possibly to real historical data will be mandatory.

Finally, the inverse classification problem could be tackled within an optimization framework. Proper optimization algorithms could be considered for the optimal selection of protective actions to apply to each considered safety-critical systems (e.g., NPP). The results can, then, be compared with the ones obtained by the sensitivity indicators proposed in the present paper.

\section{REFERENCES}

1 Kröger W, Zio E. Vulnerable Systems. UK, London: Springer, 2001.

2 Aven T. Foundations of Risk Analysis. Berlin: Wiley, N.J, 2003.

3 Aven T. Some reflections on uncertainty analysis and management. Reliability Engineering and System Safety, 2010; 95, 195-201.

4 Aven T. Misconceptions of Risk. Chichester: Wiley, 2010. $\square$

5 Aven T, Heide B. Reliability and validity of risk analysis. Reliability Engineering and System Safety, 2009; 94, 1862-1868.

6 Wang TR, Mousseau V, Zio E. A Hierarchical Decision Making Framework for Vulnerability Analysis. Proceedings of ESREL2013, Sep 2013, Amsterdam, Netherlands. pp.1-8. 
7 Leroy A, Mousseau V, Pirlot M. Learning the parameters of a multiple criteria sorting method, The Second International Conference on Algorithmic Decision Theory,Algorithmic Decision Theory, R.I. Brafman, F. Roberts, and A. Tsoukiàs (Eds.): ADT 2011, LNAI 6992, pp. 219-233, Germany, Berlin: Springer, 2011

8 Charu C, Chen C, Jiawei H. The Inverse Classification Problem, JOURNAL OF COMPUTER SCIENCE AND TECHNOLOGY 25(3): 458-468 May 2010

9 Aggarwal CC, Chen C, Jiawei H. On the Inverse Classification Problem and its Applications. Data Engineering, 2006. ICDE '06. Proceedings of the 22nd International Conference, 2006. IEEE.

10 Aiguo L, Xin Z, Jiulong Z. Performance Analysis of Quantitative Attributes Inverse Classification Problem. JOURNAL OF COMPUTERS, Vol.7, No. 5, May 2012.

11. Aven T, Flage R. Use of decision criteria based on expected values to support decisionmaking in a production assurance and safety setting. Reliability Engineering and System Safety, 2009; 94, 1491-1498.

12 Hofmann M, Kjølle G, Gjerde O. Development of indicators to monitor vulnerabilities in power systems, 2012 International Conference on Probabilistic Safety Assessment and Management (PSAM 11) \& European Safety and RELiability Conference (ESREL 2012); Helsinki, Finland.

13 NWRA, N. W. R. A. Risk assessment methods for water infrastructure systems, Rhode Island Water Resources Center, University of Rhode Island, Kingston, RI. 2012.

14. Roy B. The outranking approach and the foundations of ELECTRE methods. Theory and Decision 31, 1991, 49- 73.

15. Mousseau V., Slowinski R. Inferring an ELECTRE TRI Model from Assignment Examples. Journal of Global Optimization, vol. 12, 1998, 157-174.

16 Wang TR, Mousseau V, Pedroni N, Zio E. Assessing the confidence of a classificationbased vulnerability analysis model, Risk Analysis, doi:10.1111/risa. 12305, 2014.

17 Saltelli A, Ratto M, Andres T, Campolongo F, Carboni J, Gatelli D, Saisana M, Tarantola S. Global sensitivity analysis. The primer. Chichester: Wiley, 2008. 
18 Iooss B, Lemaître P. A review on global sensitivity analysis methods. Global sensitivity analysis, 2014

19 Saltelli A, Tarantola S, Campolongo F, Ratto M. Sensitivity analysis in practice. Chichester: Wiley, 2004

20 Rocco C, Zio E. Bootstrap-based techiniques for computing confidence intervals in monte carlo system reliability evaluation. $\square$ Reliability and Maintainability Symposium, 2005. Proceedings. Annual. Page(s): 303 - 307.

21 Levitin, G., Hausken, K., and Ben Haim, H. (2013), "Defending Majority Voting Systems Against a Strategic Attacker," Reliability Engineering \& System Safety 111, 1, 37-44.

22 Thekdi, S.A., and J.H. Lambert 2014. Quantification of scenarios and stakeholders influencing priorities for risk mitigation in infrastructure systems. ASCE Journal of Management in Engineering. 30(1):32-40.

23 Karvetski, C.W., and J.H. Lambert 2012. Evaluating deep uncertainties in strategic priority-setting with an application to facility energy investments. Systems Engineering. 15(4): 483-493.

24 Martinez, L.J., J.H. Lambert, and C. Karvetski 2011. Scenario-informed multiple criteria analysis for prioritizing investments in electricity capacity expansion. Reliability Engineering and System Safety. 96: 883-891.

25 Hamilton, M.C., J.H. Lambert, J.W. Keisler, I. Linkov, and F.M. Holcomb. 2013. Research and development priorities for energy islanding of military and industrial installations. ASCE Journal of Infrastructure Systems. 19(3):297-305.

26 Rogerson, E.C. and J.H. Lambert 2012. Prioritizing risk via several expert perspectives with application to airport runway safety. Reliability Engineering and System Safety. 103: 2234.

27 Joshi, N.N. and J.H. Lambert 2011. Diversification of engineering infrastructure investments for emergent and unknown non-systematic risks. Journal of Risk Research. 14(4): $1466-4461$. 
28 Lambert, J.H. and M.W. Farrington 2007. Cost-benefit functions for the allocation of security sensors for air contaminants. Reliability Engineering and System Safety. 92(7):930946.

29 Zio E. An Introduction to the Basics of Reliability and Risk Analysis. World Scientific Publishing Co, 2007.

30 Larsson, J.E. Knowledge-based methods for control systems. PhD dissertation, Lund Institute of Technology, Department of Automatic Control, 1992.

31 Doumpos, M. and C. Zopounidis 2002. Multricriteria Decision Aid Classification Methods, Kluwer Academic Publishers, Netherlands. $\square 2002$, ISBN 1- 4020-0805-8.

\section{APPENDIX A CLASSIFICATION MODEL FOR VULNERABILITY} ANALYSIS: THE MAJORITY RULE SORTING (MR-SORT) METHOD

The Majority Rule Sorting Model (MR-Sort) method is a simplified version of ELECTRE Tri, an outranking sorting procedure in which the assignment of an alternative to a given category is determined using a complex concordance non-discordance rule (14)(15). We assume that the alternative to be classified (in this paper, a safety- critical system or infrastructure of interests, e.g., a nuclear power plant) can be described by an n-tuple of elements $x=\left\{x_{1}, x_{2}, \ldots\right.$, $\left.x_{i}, \ldots, x_{n}\right\}$, which represent the evaluation of the alternative with respect to a set of $n$ criteria (by way of example, in the present paper the criteria used to evaluate the vulnerability of a safety critical system of interest may include its physical characteristics, social criticality, level of protection and so on: see Section 2). We denote the set of criteria by $N=\{1,2, \ldots$, $\mathrm{i}, \ldots, \mathrm{n}$ ) and assume that the values $x_{i}$ of criterion $i$ range in the set $X_{i}$ (20) (for example, in the present paper all the criteria range in $[0,1])$. The MR-Sort procedure allows assigning any alternative $x=\left\{x_{1}, x_{2}, \ldots, x_{i}, \ldots, x_{n}\right\} \in X=X_{1} \times X_{2} \times \ldots \times X_{i} \times \ldots \times X_{n}$ to a particular pre-defined category (in this paper, a class of vulnerability), in a given ordered set of categories, $\left\{\mathrm{C}^{\mathrm{h}}: \mathrm{h}=1,2, \ldots, \mathrm{M}\right\}$; as mentioned in Section $2, M=4$ categories are considered 
in this work: $A^{l}=$ satisfactory, $A^{2}=$ acceptable, $A^{3}=$ problematic, $A^{4}=$ serious .

To this aim, the model is further specialized in the following way:

- We assume that $X_{i}$ is a subset of $\mathbb{R}$ for all $i \in \mathbb{N}$ and the sub-intervals $\left(X_{i}^{1}, X_{i}^{2}, \ldots, X_{i}^{h}, \ldots, X_{i}^{M}\right)$ of $X_{i}$ are compatible with the order on the real numbers, i.e., for all $x_{i}^{1} \in X_{i}^{1}, x_{i}^{2} \in X_{i}^{2}, \ldots, x_{i}^{h} \in X_{i}^{h}, \ldots, x_{i}^{M} \in X_{i}^{M}$, we have $x_{i}^{1}>x_{i}^{2}>\ldots>x_{i}^{h}>\ldots>x_{i}^{M}$. We assume furthermore that each interval $X_{i}^{h}, h=1,2, \ldots, M-1$ has a smallest element $b_{i}^{h}$, which implies that $x_{i}^{h} \geq b_{i}^{h}>x_{i}^{h+1}$. The vector $b^{h}=\left\{b_{1}^{h}, b_{2}^{h}, \ldots, b_{i}^{h}, \ldots, b_{n}^{h}\right\}$ (containing the lower bounds of in the intervals $X_{i}^{h}$ of criteria $i=1,2, \ldots, n$ in correspondence of category $h$ ) represents the lower limit profile of category $C^{h}$.

- There is a weight $\omega_{i}$ associated with each criterion $i=1,2, \ldots, n$, quantifying the relative importance of criterion $i$ in the vulnerability assessment process; notice that the weights are normalized such that $\sum_{i=1}^{n} \omega_{i}=1$.

In this framework, a given alternative $x=\left\{x_{1}, x_{2}, \ldots, x_{i}, \ldots, x_{n}\right\}$ is assigned to category $C^{h}, h=2,3, \ldots, M-1$, iff

$$
\sum_{i \in \mathbb{N}: x_{i} \geq b_{i}^{h}} \omega_{i} \geq \lambda \text { and } \sum_{i \in \mathbb{N}: x_{i} \geq b_{i}^{h-1}} \omega_{i}<\lambda,
$$

where $\lambda$ is a threshold $(0 \leq \lambda \leq 1$, e.g., in this paper $\lambda=0.9)$ chosen by the analyst. Rule (A.1) is interpreted as follows. An alternative $x$ belongs to category $C^{h}$ if: 1) its evaluations in correspondence of the $\mathrm{n}$ criteria (i.e., the values $\left.\left\{x_{1}, x_{2}, \ldots, x_{i}, \ldots, x_{n}\right\}\right)$ are at least as good as $b_{i}^{h}$ ( lower limit of category Ah with respect to criterion $i$ ), $i=1,2, \ldots, n$, on a subset of criteria that has sufficient importance (in other words, on a subset of criteria that has a weight larger than or equal to the threshold $\lambda$ chosen by the analyst); and at the same time 2) the weight of the subset of criteria on which the evaluations $\left\{x_{1}, x_{2}, \ldots, x_{i}, \ldots, x_{n}\right\}$ are at least as good as $b_{i}^{h-1}$ (lower limit of the successive category $C^{h-1}$ with respect to criterion $i), i=1,2, \ldots, n$, is not sufficient to justify the assignment of $x$ to the successive category $C^{h-1}$.

Notice that alternative $x$ is assigned to the best category $C^{1} \sum_{i \in \mathbb{N}: x_{i} \geq b_{i}^{1}} \omega_{i} \geq \lambda$ and it 
is assigned to the worst category $C^{M}$ if $\sum_{i \in \mathbb{N}: x_{i} \geq b_{i}^{M-1}} \omega_{i}<\lambda$. Finally, it is straightforward to notice that the parameters of such a model are the $(M-1) \cdot n$ lower limit profiles ( $n$ limits for each of the $M-1$ categories), the $\mathrm{n}$ weights of the criteria $\omega_{1}, \omega_{2}, \ldots, \omega_{i}, \ldots, \omega_{n}$, and the threshold $\lambda$, for a total of $(n \cdot M+1)$ parameters. 